\title{
A New approach to Designing Lateral Collision Warning Systems
}

\author{
Daniel P. Jenkins, Neville A. Stanton, Guy H. Walker \& Mark S. \\ Young
}

School of Engineering \& Design, Brunel University, Uxbridge, Middlesex, UB8 3PH, UK

\begin{abstract}
According to the National Highway Traffic Safety Administration (NHTSA), more than $43 \%$ of all fatal accidents reported in 2001 involved a lane or road departure. There are a number of systems on the market that monitor driver lane position or use radar technology to track the position of other drivers. The majority of these systems and available research focuses on the prevention of longitudinal collision or lane departures. In this paper we will discuss a system that reduces the likelihood of a lateral collision. The problem was analysed by a Work Domain Analysis, the first phase of Cognitive Work Analysis. This analysis informed an interface design that provides the driver with information on the position of other road users; and delivers a time critical warning when the driver is in a position of impending collision.
\end{abstract}

Keywords: Collision avoidance; Lateral; Lane departure; CWA; WDA, Warnings; Safety; Driving Simulator;

Reference to this paper should be made as follows: Jenkins, D.P., Stanton, N.A., Walker, G.H., \& Young, M.S. (200x)' Field of safe travel: A new approach to Designing Lateral Collision Warning Systems, Vol. X, No. Y,pp.000-000.

Biographical Notes: Dan Jenkins Graduated in 2004 from Brunel University with an M.Eng (Hons) in Mechanical Engineering and Design receiving the 'University Prize' for top student in the department. With over two years experienced as a Design Engineer in the Automotive Industry, Dan has worked in a number of roles throughout the world. This wide range of placements has developed experience encompassing; design, engineering, project management and commercial awareness. Dan is currently a full-time research fellow at Brunel University and is studying for a PhD related to Ecological Interface Design

\section{Introduction}

This paper introduces a warning system for automotive lateral collision. The paper describes the current problem and documents the design of a number of solutions. These solutions are then tested in a driving simulator environment and conclusions drawn.

The literature on lane departure and collision avoidance systems is dominated by papers on engineering developments. These articles tend to focus on the development of the technologies and the algorithms behind the systems (Barickman \& Stoltzfus (1999); Batavia (1999); Hamilton at al (2001); Hoare et al (2002); Lee et al (1999); Prakah-Asante et al $(2003,2002))$. There are also a number of papers that tackle lane departures exclusively (Suzuki and Jansson, 2003, Rudin-Brown and Noy, 2002). However there appears to be no available literature that approaches the design of lateral warning systems from a human factors perspective. In such a time critical domain it is important that any information provided to the driver can be rapidly interpreted and understood by the driver. According to Sukathankar (1997) the task of driving is complex and requires the driver to keep track of a number of critical variables in a dynamic and changeable environment. These include: their route, their position, their speed, the position and speed of other vehicles, road and weather conditions, and the behaviour of their own vehicle. Gugerty (1997), states that drivers also need to be able to predict how these variables will change in the near future, in order to anticipate how to adapt their own driving. Tilin (2002), comments that the introduction of components in the car such as phones and satellite navigation systems has increased the complexity of the driving task. According to Allen 
(2004) the main issue to be resolved in collision avoidance is the driver/system interface; this includes the driver's reaction to and effective integration within the overall vehicle system.

\section{The interface design}

The first stage of this interface design was to understand the environment lateral collisions are taking place within. One suitable framework for this is the first phase of Cognitive Work analysis, Work Domain Analysis (WDA) (Naikar et al, 2005, Burns \& Hajdukiewicz, 2004, Vicente 1999). The WDA is constructed by understanding the purpose of the system and the constraints forced upon it. The first stage of the analysis is to define the boundary of the domain under investigation. In this case the task of driving has been limited to multilane unidirectional motorway driving. The current analysis boundary does not include lane departures resulting from drivers departing the road due to improper speed, or from sudden mechanical failure such as a tire blow out.

In this analysis the overall purpose of the system can be defined as to reduce lateral collisions in motorway driving'. The analysis starts by looking at the possible ways that a collision could occur within the specified domain. Figure 1shows three possible causes of a lateral collision; the driver being unaware that they are leaving their lane and colliding with another vehicle; the driver intentionally leaving the lane at an unsafe time; and another vehicle entering the driver's lane at an unsafe time (both intentionally and unintentionally). These can be seen graphically in Figure 1 where the lighter car represents the driver and the darker car represents another road user.

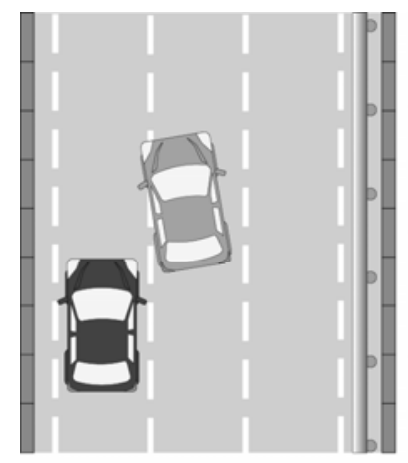

Unintended Lane Departure

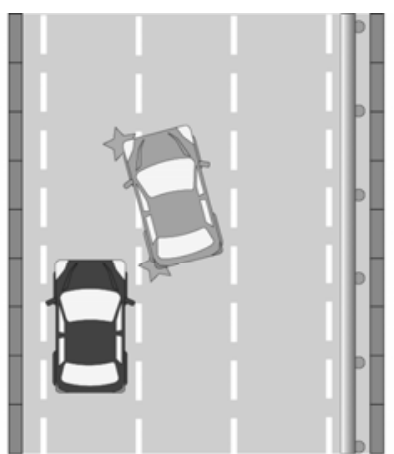

Unsafe Intended Lane Departure

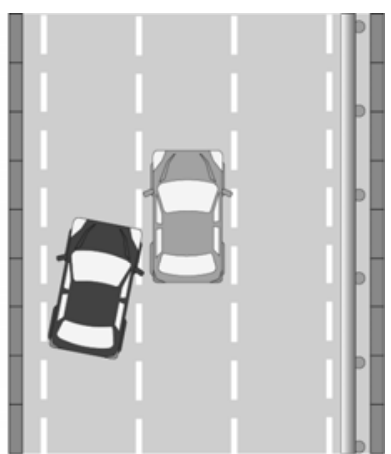

Other Vehicle Entering Lane

Figure 1 - Causes of lateral collisions

Unintentional lane departures are a result of the driver neglecting to carry out the tracking task of keeping the vehicle in between the lane markings. One of the main causes for this is fatigue with ROSPA (2001) reporting that driver fatigue could be the cause of up to twenty percent of accidents on monotonous roads in the UK. The American Automobile Association describes accidents resulting from distracted drivers as one of the most serious public health hazards of the $21^{\text {st }}$ century. Collisions resulting from intentional lane departure are due to errors in judgement; the driver has consciously decided to enter another lane; as a result of this decision the driver may have entered the path of a vehicle. Reason (1990) would class this error as a mistake; the action was intended but the outcome was not desired (i.e. potential conflict with adjacent vehicle(s)); the reasons for a mistake are numerous, an example of this could be due to the drivers not checking their blind spot (i.e. a lapse). The third type of collision shown shows another vehicle entering the drivers. A collision will result if the driver fails to see the vehicle enter their lane or the driver is unable to take appropriate evasive action in time.

Figure 2 shows the WDA for the specified domain; starting with the top level the 'Functional Purpose' describes the system's raison d'être. At the bottom level the 'Physical Objects' list the existing technologies in mass production cars that can be used to provide the driver with information, in the level above this titled 'object related processes' the functions that the objects can afford are listed. The level in the centre of the diagram lists the functions that can be afforded by the physical objects specific to the context of reducing lateral collisions. In 'values and priority measures' the criteria against which the function purpose can be 
assessed are included. Once the diagram is completed means ends links are created between the nodes in the diagram.

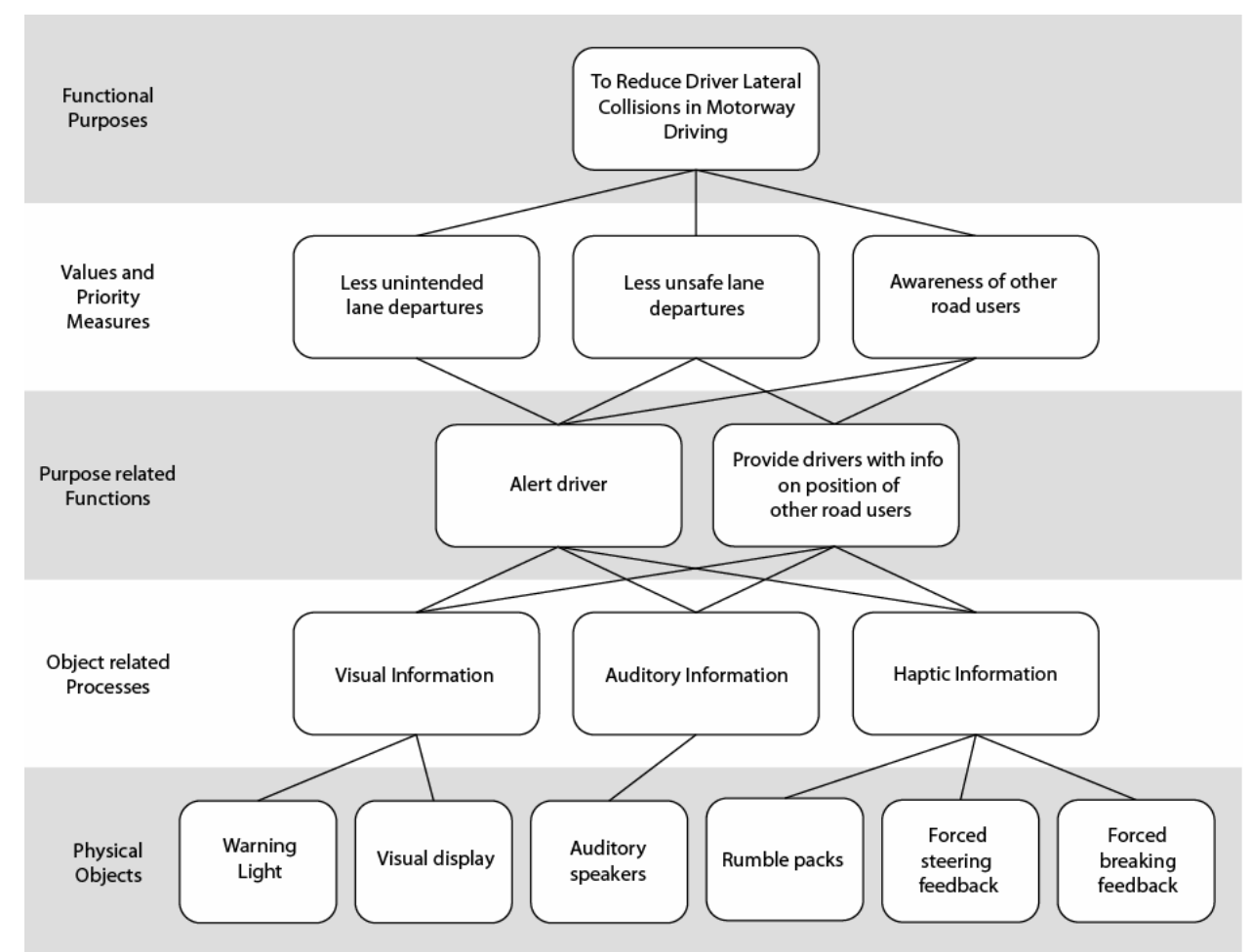

Figure 2 - WDA

The means-end relationships shown in Figure 2 show that in order to warn the driver against the three types of collision a function is required to alert the driver. The diagram also shows that providing the driver with positional information is not necessary in unintended lane departures as if the driver is unaware of their departure they would not be able to correct against it.

\subsection{Feedback mechanisms}

A number of feedback methods have been previously investigated in the field of collision avoidance. These can be classified by the modes of communication that they apply to, fitting them into the object related processes level of the WDA

Audio alarm - The audio alarm is one of the most common alarms, it is used in a wide range of applications. The main benefits of an audio alarm are that the user doesn't have to be focusing on a display or even the task. The selection of the type of audio alarm is very important; it needs to be of the right pitch, tone and volume to be heard above the other noises in the car, without being so loud that it startles and panics the driver. The driver also needs to be able to relate the sound to the problem causing the alarm. In a driving situation temporal performance is imperative; an indistinguishable alarm could be more of a hindrance and distract the driver's attention away from correcting the vehicle's course. The alarm can be connected to the by a semantic link; one method used to achieve this is by using a sound that the driver already associates with leaving the lane such as the noise generated by rumble strips. Rumble strips are areas of grooved pavement or grooved lane markings. When the vehicle leaves the lane, the tyres hit the rumble strip and thereby produce a noise which alerts the driver. Extensive simulator tests at the DaimlerBenz Driving Simulator and on-the-road experiments into lane departure warnings in the trucking industry by Ziegler (1995) showed that when compared against abstract warning tones. A recording of rumble strips resulted in greater driver acceptance and faster reactions.

Tactile - Pohl \& Ekmark (2003) identified that [audio] false alarms can be considered by the driver as patronising and consequently annoying. Many of the current warning systems have been designed for the 
trucking industry and have generally used auditory alarms to signify an unintentional lane departure. The significant difference between the trucker and the personal car driver is that the trucker generally drives alone where as the personal car owner is likely to transport passengers. Because of this a personal car owner may have a much lower acceptance of a system that alerts the whole car and may seek a feedback method that only the driver can sense. The use of rumble packs within the seat is one way of achieving this. Another way of feeding back information is through the steering wheel. The amount of torque applied to the steering wheel as feedback can be varied as an indication of how much to correct the wheel. Haptic braking is another way of simulating the vibration feedback of rumble strip. The system rapidly applies and releases the brakes creating a juddering vibration in the car similar to that of running over the bumpy surface of the rumble strips.

Visual displays - The introduction of computer screens into the driving environment has become far more common in recent years; most car radios now contain a computerised graphic display showing progressively more information. An increasingly popular way of monitoring a screen while conducting another visual task is in the use of head up displays. The advantages of head up displays are primarily based around the fact that the driver does not have to keep changing their focal range - this is done by moving the important information a driver needs to see up into their line of sight, so they don't have to take their eyes off the road as the image appears to float in mid air, just past the front end of the vehicle. Head up displays have proved successful in the aviation domain where because of the high closing speeds involved if you've seen it outside the window it's probably too late anyway. The aviation domain is however very different to driving with far less visual clutter. Whether the advantages of this transfer as readily to such a visually dominant task as driving (where if you've seen it you can probably avoid it) are not yet fully resolved (e.g. Tufano, 1997).

\subsection{Multimodal feedback}

Multimodal feedback provides feedback to two or more of the feedback mechanisms (audio, tactile, visual). Clarke (2003), reports that when compared against single mode feedback a well matched combination of feedback modes is optimum. The main drawback to multiple feedback methods is the cost and the possibility that they may startle or confuse the driver if the modes are poorly matched. Wheatley (2000) states that cross modal information presentation will add a new dimension of complexity with certain types of alarm mixing better than others. It has been shown that both auditory and tactile alarms mix well with visual warnings; however when tactile and auditory are combined reaction times were shown to be slower than the two components independently.

The WDA shows that there are two separate purposes of the system these are to alert the driver and to provide the driver with information on the position of other road users. As these requirements are different it is also likely that the feedback method required may also be different. The purpose of 'alerting the driver' is designed to prompt the driver when they may be distracted, for this reason the warning needs to be very simple signal indicating either a safe or a dangerous state. The simplicity of the warning may affect the driver's ability to respond to it. Whilst looking at warnings originating from speakers around a room Wallace et al (1996) found that the reaction time increased with the number of speakers (400 ms for 1 speaker to 800 ms for 6 speakers). Wallace et al also report that participants were quicker to respond to tones presented directly in front of them.

In order to develop each of these solutions further an investigation was conducted. Two studies were conducted to evaluate a number of generated solutions the initial study recording driver reaction time and subjective opinion was conducted on a PC based program. A follow up study was conducted in the Brunel University driving simulator to investigate a short list of displayed in a more naturalistic setting.

\section{STUDY 1 - Selection of Warnings}

\subsection{Evaluating the appropriateness of the feedback methods.}

Seven sounds were selected to cover a range of sound types (Metaphoric, naturalistic \& abstract). These included; the sound of a car skidding; a smoke detector bleeping; a car horn; a truck horn; a car alarm; a car running over rumble strips; and a European siren. 
Seven pictorial displays were also selected; the starting point in the design of these displays was the idea of informing the driver of the area where it is safe to position the vehicle. This concept was first conceived by Gibson \& Crooks, (1938)

"Within the boundaries of the road lies, according to our hypothesis, an indefinitely bounded field which we shall name the field of safe travel. It consists, at any given moment, of the field of possible paths which the car may take unimpeded. Phenomenally, it is a sort of tongue protruding forward along the road. Its boundaries are chiefly determined by objects or features of the terrain with a negative 'valence' in perception." (Gibson \& Crooks, 1938, pp. 454).

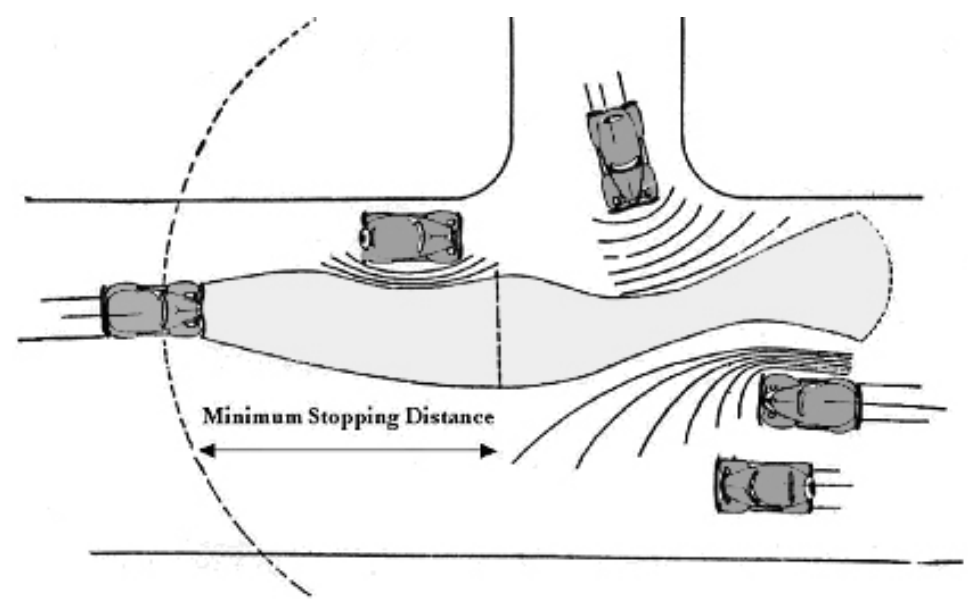

Figure 3 Diagram showing illustration of the field of safe travel. (Adapted from Gibson \& Crooks, 1938) 
Table 1

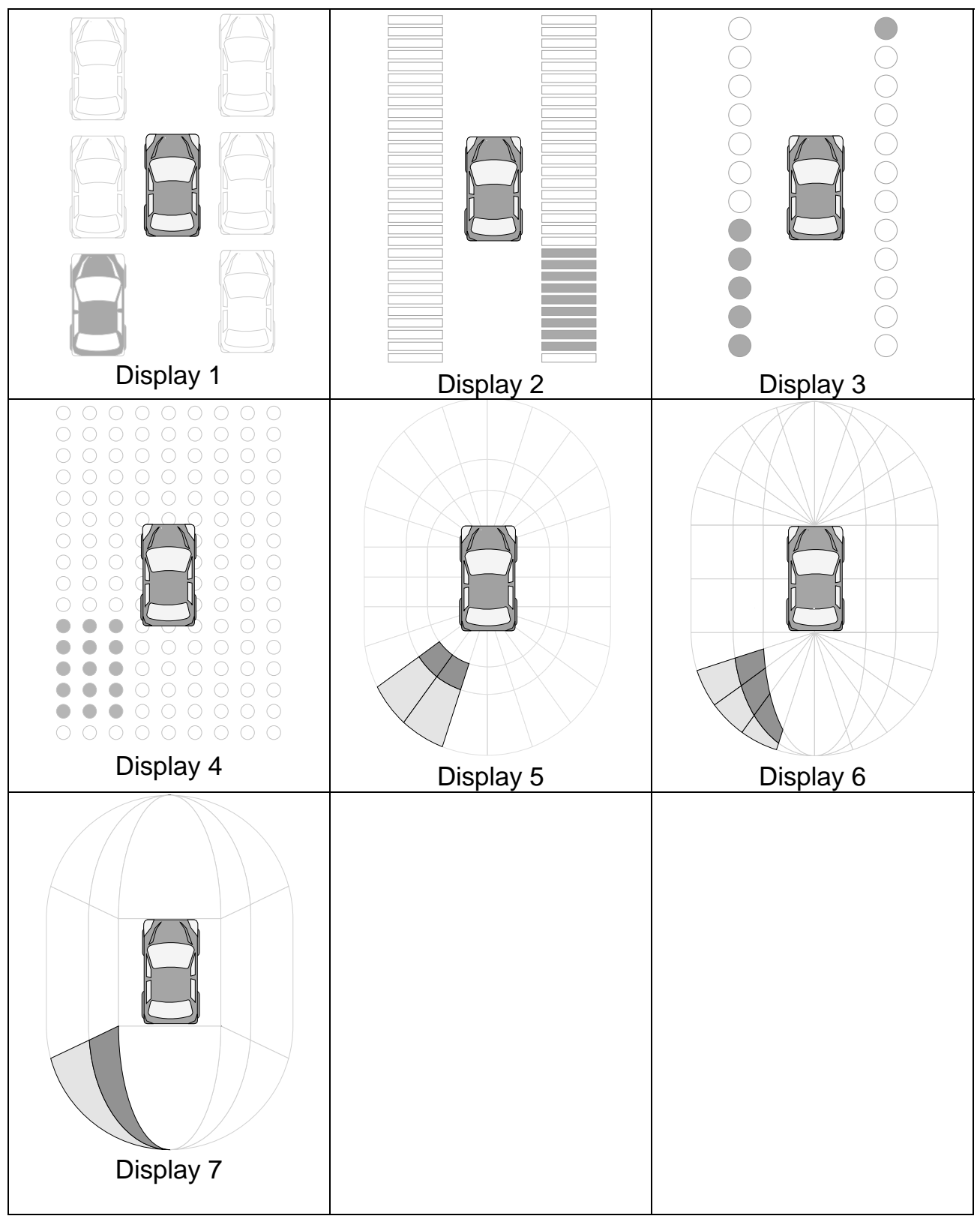

Display 1 has been designed so that its function is easily recognisable; the display is very pictorial to make it easier to understand what the display is for. The display shows the possible locations for a warning in a wire-frame. When an object is detected at this location the wire frame car is filled in. Display 1 shows a vehicle present to the rear left of the car.

Display 2 has been designed to more accurately show the position of the vehicle. Display 1 was limited to only 6 possible positions that the vehicle could be located; Display 2 allows a much more accurate positioning. The display deliberately avoids the use of a car like representation to prevent the driver from assuming the vehicle is a car when it could also represent trucks, cyclist, or motorcyclists. The length of the vehicle can also be changed with this display. The example shown shows a vehicle to the rear right of the car.

Display 3 shows a similar solution to Display 2 using circles in the place of boxes in an attempt to avoid confusion with other displays in the car such as fuel gages. Here two vehicles are shown a vehicle to the rear left and a vehicle ahead to the right. 
The display shown in Display 4 has more combinations which possibly makes it harder to scan. The advantage of this display however is that lateral position can also be established. Here a vehicle is shown that appears to be the same size as a car to the rear left.

Display 5 has been developed based on a field of safe travel. This solution builds on the work of Gibson \& Crooks (1938). The ring highlights the limit of the sensing ability. It also attempts to show how the system is working in order to assist the driver in constructing a correct mental model. The addition of the three colour states (red amber and yellow) show the driver a level of significance of the location

Display 6 shows a more accurate representation of the safe and dangerous areas than Display 5 . It is not really suitable to use a universal distance ring as more space has to be left in the longitudinal direction than the lateral.

Display 7 shows a simplified version of Display 6 sacrificing some of the accuracy in placement of the object in order to make the display easier to scan.

\subsection{Experimental methods}

The purpose of the first experiment was to limit the generated solutions down to a short list of the three most appropriate sounds three most appropriate visual displays. A PC based computer program was developed to gain participant reaction times and subjective rating before using the driving simulator in the second experiment.

\subsection{Participants:}

An unstratified sample of 9 participants was selected to take part in the initial trial 7 male and 2 female, with a mean age of $24.9(S D=14.08)$. The decision was taken not to stratify the sample in order to keep the required number of participants to a minimum.

\subsection{Design:}

A within subject study was employed with three dependent variables relating to driving performance. These were Reaction time (measured in milliseconds); appropriateness and urgency (measured using a self report questionnaire and likert scales). Two independent feedback variables were manipulated; these were audio and visual with 7 levels (detailed earlier in this report). A two-tailed prediction denoted that there might be differences in the reaction time and level of appropriateness when feedback is varied. Furthermore a twotailed prediction suggested that the ratings of urgency would differ once audio feedback was varied. Each of the trials lasted approximately 24 minutes. The participants were asked to complete 14 short computer programs (one for each of the generated solutions).

\subsection{Equipment:}

This study took place in a small environmentally controllable room. The programs were run on a standard desktop computer (PC Pentium 4; 1.7GHz; 256 MB RAM) connected to a 2 way speaker system with bass box. Macromedia flash was used to create and run the programs in which reaction time was automatically captured. Participant subjective feedback was captured on hardcopy questionnaires.

\subsection{Procedure:}

The 7 Auditory and 7 visual displays were presented independently in the same way. At the start of each experiment participants were presented with a screen with no warnings present, when a warning was presented the participant had to identify the direction (left or right) via a key press; the software recorded the participant's reaction time. The order of presentation of graphical and visual experiments was randomised. The procedure was as follows:

1. Participant were welcomed and introduced to main aims of the trial

2. Participants were asked to complete a short practice program for a unique graphical display.

3. The participants were offered the opportunity to pose further questions 
4. The participants tackled each of the graphical solutions in a random order

5. After each test was completed the results were recorded along with the participant's subjective rating of appropriateness

6. Participants completed the practice solution for a unique auditory response

7. The participants tackled each of the auditory solutions in a random order

8. After each test was completed the results were recorded along with the participant's subjective opinion of urgency and appropriateness.

9. The participants were thanked for their participation and dismissed.

\section{Results and Discussion: Icon selection experiment.}

Participant reacted faster to the auditory icons than the visual icons.

Table 2

\begin{tabular}{|l|l|l|}
\hline & Visual & Auditory \\
\hline Mean & 0.61 & 0.47 \\
\hline Standard Deviation & 0.165 & 0.115 \\
\hline
\end{tabular}

For both the audio and the visual icons a decision matrix was employed to select the optimum solution by weighting the importance of the factors, (reaction time, appropriateness rating and urgency rating).

In order to determine the optimum solution first the relative importance of each of independent variable was established. In the case of the visual display the rating of the reaction time is not critical especially as the results are all so close. What is far more important is the driver's acceptance of the display which can be inferred by the appropriateness rating. For this reason an $80-20$ split in the favour of the appropriateness rating was assigned. A decision matrix was employed to calculate a final score for each of the independent variables. The decision matrix works by taking the original score and multiplying it by the weighting ratio to give a new number. These new numbers are then summed to give a score out of ten for each of the icons examined. The displays marked with an $\mathrm{X}$ were selected to be carried forward to the next stage of testing.

\begin{tabular}{|c|c|c|c|}
\hline & & 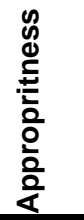 & 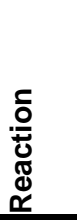 \\
\hline V1 & \multirow{7}{*}{$\begin{array}{l}\text { Radar real } \\
\text { Cars } \\
\text { Radar large } \\
\text { Radar geo } \\
\text { Dots many } \\
\text { Squares } \\
\text { Dots lines }\end{array}$} & 8.67 & 5.08 \\
\hline V2 & & 8.00 & 4.76 \\
\hline V3 & & 6.89 & 4.29 \\
\hline V4 & & 8.44 & 5.56 \\
\hline V5 & & 7.11 & 5.95 \\
\hline V6 & & 7.56 & 7.30 \\
\hline V7 & & 7.33 & 7.06 \\
\hline
\end{tabular}
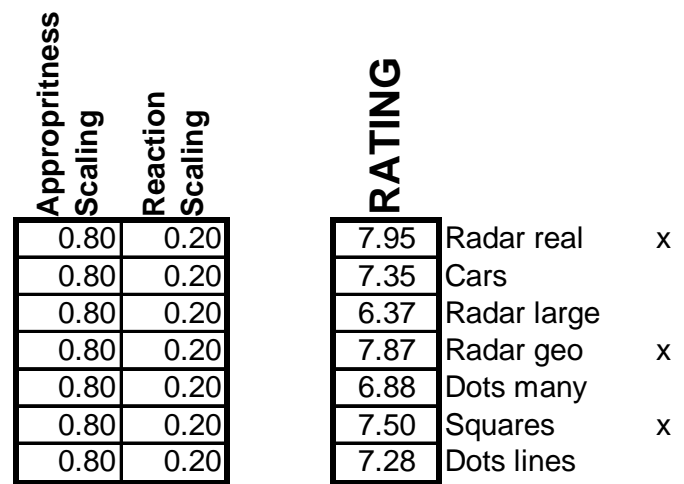

Figure 4 


\section{Figure 5}
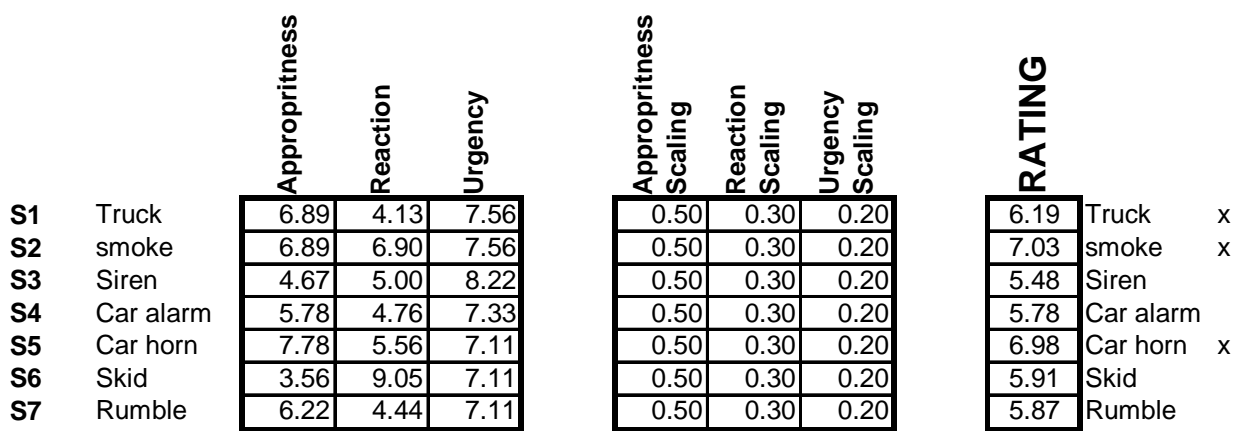

The generic issue of the sounds being confused with in car entertainment was frequently addressed by the participants. It may therefore be important to mute the in car entertainment or to play the sound through a different set of speakers. There seemed to be mixed opinion on the type of sound that should be used; some participants preferring the iconic sounds of the horn as they created semantic links with cause. Other participants found that these iconic sounds could be too easily confused with background noise and preferred the arbitrary bleeps that they associated with a warning.

The driver's ability to interpret and react to the auditory signal is of paramount importance. At a first glance it may therefore appear that the rating should be based purely on the reaction time, however it must be considered that this experiment was conducted outside the environment and the participants were tested purely on their ability to react to the sound and determine its direction. The cognitive level of selecting what the sound meant and what the warning implied was completely removed. There is no point in the driver being able to understand a signal very quickly if they do not interpret what the signal means. For this reason the appropriateness rating and the urgency rating also need to be included in the decision matrix.

It is important that the displays are considered in their natural environment as the initial testing allowed the participants to evaluate the tasks using their entire mental capabilities. In the real life situation the drivers will only have a percentage of their cognitive ability to devote to the task as part of it will be taken up completing the driving task. It is highly likely that this will affect the way in which the participants react with the feedback.

\section{STUDY 2 - Driving Simulator Experiment}

In order to determine which of the short listed displays and sounds was most appropriate the solutions were evaluated in the Brunel University driving simulator environment.

\subsection{Participants:}

12 participants were recruited, all of whom were male with a mean age of $23.4(S D=2.27)$ and participants were unstratified.

\subsection{Design:}

In order to asses the suitability of each of the feedback methods, it was desirable to capture both the participant's reaction time to respond to the warning as well as their subjective rating. Reaction times were recorded using an adaptation of the flash program developed for the initial participant feedback in the first stage of the trials. Left and right push buttons attached to the simulator vehicle's steering wheel interfaced with the computer and served as inputs to the flash program.

As with experiment 1, after the warning had been presented 5 times the experiment was stopped and the participant asked to rate the warning on simple Likert scales against appropriateness. Feedback on the perceived urgency was also recorded on Likert scales for the auditory warnings. 


\subsection{Equipment:}

Experiment 2 took place in the Brunel University driving simulator suite which comprises a full Ford Mondeo positioned in front of a projected road image. The software for the simulator is run on a standard desktop PC (Tri Gem - Gem Master P90 PC) connected to 'Logic3 ScreenBeat 1P' 10 Watt Speakers. Warnings were presented using flash programs (3 visual and 3 audio programs).

\subsection{Procedure:}

1. Participant welcomed and introduced to main aims of the trial

2. Participant details recorded (gender, age, driving experience, type of vehicle owned and average mileage)

3. The function of the car and the driving simulator environment was explained to the participant.

4. The driver was then asked to complete a short practice drive in the simulator environment. Each participant was asked to maintain a constant speed and drive the car for 5 minutes to acclimatise themselves with the new environment.

5. The participant was asked to carry out a practice test to ensure they understood how to respond when presented with a warning.

6. The experiments were presented in a random order; the participant was given a short break in between tests, where their subjective feedback was recorded.

7. The participant was thanked for their participation and dismissed.

\subsection{Results and discussion:}

The results of a one-way ANOVA show that there are statistically significantly different between reaction time for the two warnings modalities (auditory and visual) $\left(F_{5,66}=177.9, p<0.01\right)$. A Scheffe post hoc test was then conducted, each of the graphical displays were all significantly slower than each of the auditory warnings $(p<0.01)$.

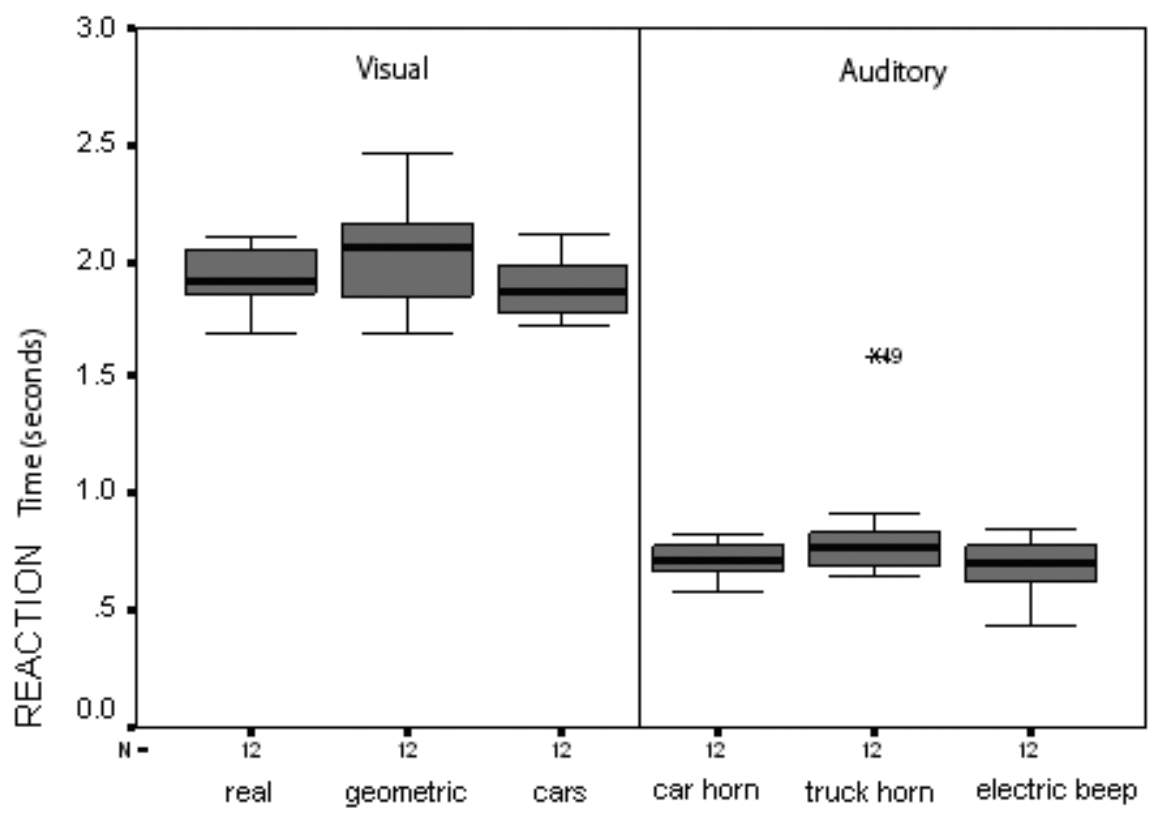

Figure 6

The results of a one-way ANOVA for appropriateness show that there are no statistically significantly differences between the warnings. $\left(F_{5,66}=1.305, p=n s\right)$.

The results of a one-way ANOVA for urgency show that there are statistically significantly differences between the warnings. $\left(F_{5,66}=3.314, p<0.01\right)$. The post hoc test however was inconclusive. 


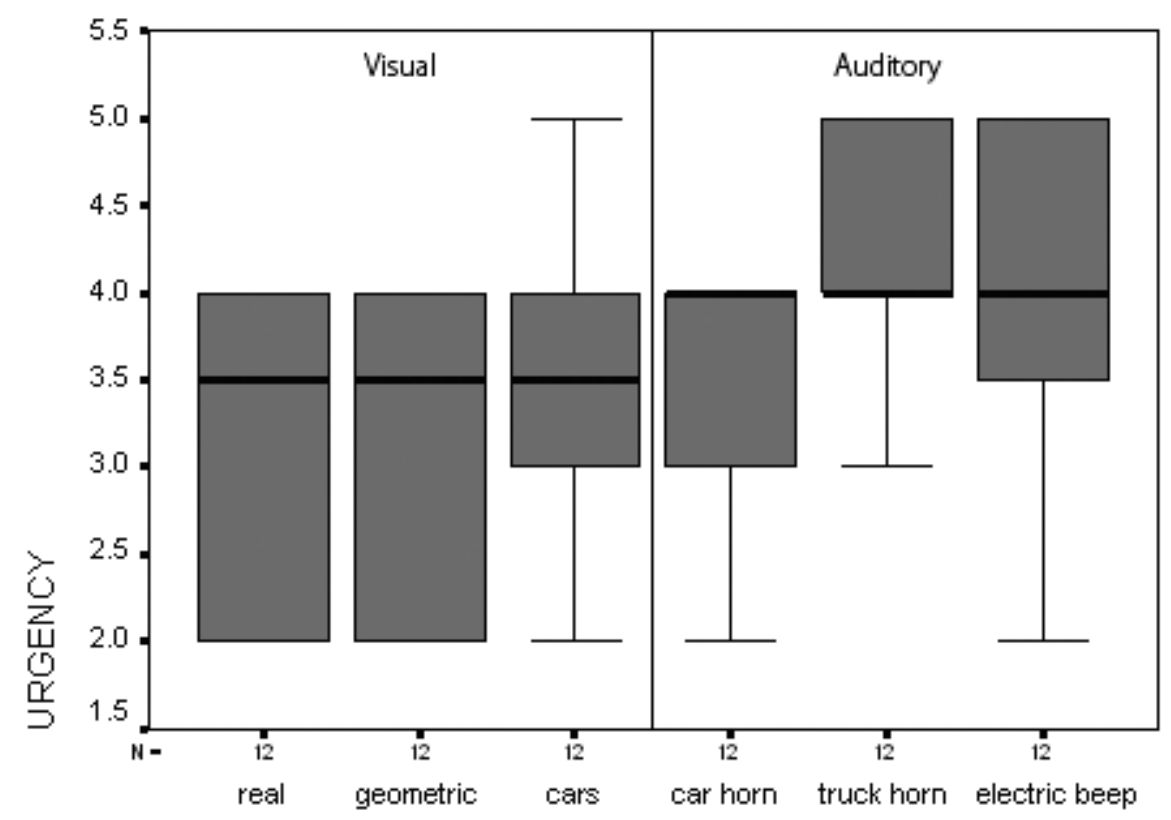

Figure 7

When these times are considered as a distance the ecological as opposed to purely statistical significance is further highlighted. The participants were instructed to run the simulator trial at an average speed of $70 \mathrm{mph}$, this equates to $31.3 \mathrm{~m} / \mathrm{s}$. It can be seen in Figure 8 that with the visual response the car would have travelled an additional 37.8 meters - this equates to over 9 car lengths (Based on a Ford Focus of length $4174 \mathrm{~mm}$ travelling at a speed of $70 \mathrm{mph}$ ).

It is important to also remember that this is not the stopping time; it is purely the reaction time to the stimuli. In a real life situation the time it takes the vehicle to come to rest after the break is actuated would also be a factor; however this would be constant and irrespective of whether the warning was auditory or graphical.

Figure 8

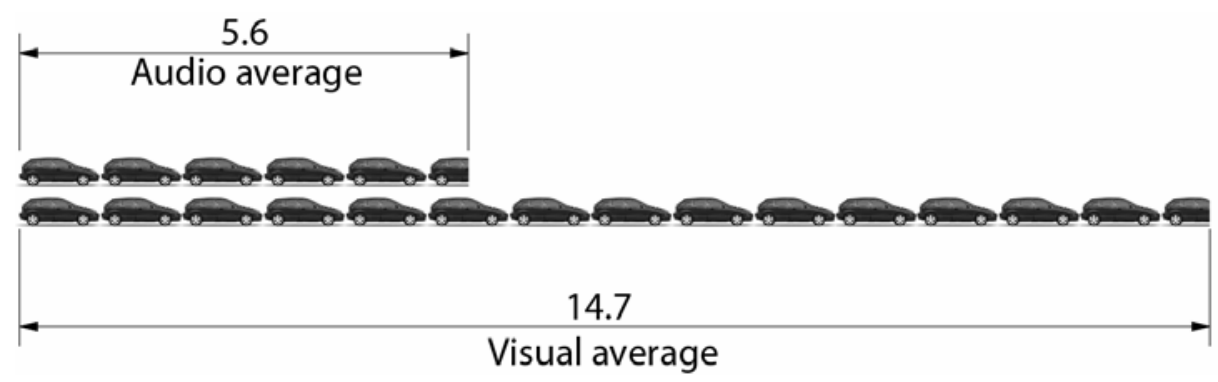

Participants were much slower at reacting to the pictorial display when compared with auditory warnings making pictorial displays unsuitable in very time dependent situations. The average reaction time for a visual display was more than twice that of an auditory display. Because the driver is unable to devote all of their attention to a graphical display a purely visual based system inappropriate. The visual display is far less intrusive than its auditory counterpart making it far more suited to the task of raising driver awareness and the development of a mental model in non critical situations. It can therefore be argued that a two stage, multi modal warning based on situational criticality would be a rational basis from which to proceed with a full scale system. The visual display would be placed towards the primary safety end of the continuum, aiding driver to gain information on the environment. The auditory warning is placed much more towards the secondary safety end of the continuum, occurring as it does in the moments before an action leads to an incident. This use of dual modality is supported by the NHTSA who comment that auditory signals should be 
reserved for more urgent warnings. This decision is also supported by Pohl and Ekmark (2003) who comment on the problems arising from frequent auditory warnings in the domestic automobile and poor driver acceptance of these systems.

\subsection{The visual feedback}

Instant recognition of the meaning of the pictorial display is less important than it is for the auditory icon. The pictorial display is used in situations where the presence of a potential hazard is less important. The purpose of the display is not intended as a warning function; its primary role is for monitoring and providing environmental information. By receiving information on the position of the hazards on the display and correlating this to the real world even an unfamiliar driver should be able to infer the purpose of the display and create an accurate mental model. This interpretation and understanding allows the driver's level of trust and confidence in the system to increase.

\subsection{The auditory feedback}

An important consideration to make about the icons selected for the auditory sound and their appropriateness is that the rating given by the participants does not really consider the ability to determine the meaning of the sound. The participants were explained the function of the testing and therefore knew that the sound was a lateral collision warning. It has been established that one of the most important functions of the sound is that it informs the driver what the root cause of the alarm is. As this inference test could not be included, it is important that the drivers interpretation of the sound needs to be considered along side the numerical results.

The NHTSA make the point that the sound should be clearly distinguishable from any other sound in the cab (car in this case). They make no reference to confusion with external sounds, however it should be inferred that confusion should not occur with common sounds originating outside the vehicle that enter the driving environment.

The primary role of the auditory icon is to regain the driver's attention and to draw it to the display. The sounds should be set up so that it appeared to originate from the graphical display thus drawing the driver's attention to the area that needs to be focused on. The other main advantage of having a unidirectional sound is that according to (Wallace et al, 1996) it is far easier to respond to.

\section{Conclusion}

\subsection{Comparison of the system against Lane departure systems}

The collision warning system (CWS) proposed in this document can be compared against existing camera based lane departure warning systems (LDW).

Table 3 shows a number of situations pictorially and comments as to whether an auditory warning would be generated. The grey car represents the car fitted with the system. The black car represents another vehicle. Each of the common situations is presented in turn; a tick indicates that a warning would be presented; a cross indicated that a warning would not be presented. These responses are circled if the outcome is undesirable.

It can be clearly seen in Table 5 that the existing lane tracking systems are presenting undesirable warnings in three of the six situations (situations 3, 4 and 6). The LDW system does not allow the driver to make safe un-indicated lane changes (situation 3). This is important as it relates to entirely appropriate forms of driver behaviour. The Highway Code (1993) states that the driver does not need to indicate if alone on the road. The Police Driver's System of Car Control reinforces the point by advising the motorist "to give a signal only when pedestrians or other road users could benefit" (Coyne, 1994, p.95). Linking the LDW to the indicator, therefore, leads to a massive increase in false alarms and an increase in customer dissatisfaction. More worryingly the system does not send a warning if the driver indicates and enters an occupied lane (situation 4), yet this is the most common road accident for vehicles travelling in the same direction according to the NHTSA. The LDW also fails to send a warning if another vehicle enters the lane occupied by the driver (situation 6). This is because the system purely tracks the lane markings and is unaware of the movements of other road vehicles and obstacles. Overall then, it can be seen that the 
behaviour of current LDW devices is at what Norman (1990) would term an intermediate level of intelligence. Intelligent enough to warn the driver in some mundane instances, but not of sufficient intelligence to assist the driver in more challenging or unambiguous circumstances, exactly those where it should be most help.

The final generated solution is unique and fundamentally different from lane departure warning systems as it also warns against intentional unsafe lane changes. By monitoring other drivers as apposed to the lane markings it also provides a warning of other drivers who move into the 'safe zone' of the car fitted with the system. 
Table 3

\begin{tabular}{|l|l|l|l|}
\hline Situation & CWS & LDW \\
\hline
\end{tabular}

The acceptance of the system is a complex issue it is understood that this technology and way of driving the car proposes a significant change and great leaps in technological change are often slow in its acceptance. This proposed solution may therefore have to be introduced in stages or introduced into the market through a niche product aimed at a population group that has high acceptance for new technology. In a similar study Bishop and Jackson (2001) state that, while only $43 \%$ of the drivers surveyed would 
purchase an ACC (adaptive cruise control) system, $98 \%$ of drivers who actually drove with an ACC said they would purchase the system.

\section{References}

Allen, R. W., (2004) The driver's role in connection with collision avoidance and advanced technology systems. Advances in Transportation Studies an international Journal Section A2

Barickman, F. S., \& Stoltzfus, D. L., (1999) A Simple CCD Based Lane Tracking System. Society of Automotive Engineers Technical paper 1999-01-1302

Belz, S.; Robinson, G. S. \& Casali, J. G. (1998) Auditory icons as impending collision warning signals in commercial motor vehicles. Proceedings of the Human Factors and Ergonomics Society 42nd Annual Meeting (pp 11271131). Santa Monica: HFES.

Bishel, R., Coleman, J., Lorenz, R., Mehring, S., (1998) Lane Departure Warning for CVO in the USA. Society of Automotive Engineers Technical paper 982779

Bishop, J. H., \& Jackson, L. E., (2001) National Transportation Safety Board Accident Investigations and Recommendations on Technologies to Prevent Rear-End Collisions. Society of Automotive Engineers Technical paper 2001-01-3243

Burns, C.M., \& Hajdukiewicz, J.R. (2004). Ecological Interface Design. Boca Raton, FL: CRC Press.

Clarke, N. J., (2003) Anti-Collision Autonomous Support and Safety Intervention System Report on Users' Needs. Jaguar internal report

Coyne, P. (1994). Roadcraft: The police driver's handbook. London. HMSO.

Dingus, T. A., McGehee, D. V., Manakkal, N., Jahns, S. K., Carney, C., Hankey, J. M., (1997). Human Factors Field Evaluation of Automotive Headway Maintenance/Collision Warning Devices, Human Factors, Vol. 39(2) p 216-229

Endsley, M., R. (1995). Towards a theory of Situation Awareness in Dynamic Systems, Human Factors, Vol. 37, pp 3264.

Gibson \& Crooks, (1938) Field of safe travel. American Journal of Psychology 51: 453-471

Gugerty, L. J., (1997) Situation awareness during driving: Explicit and implicit knowledge in dynamic spatial memory Journal Of Experimental Psychology: Applied 3 (1) 42-66

Hamilton, L., Humm, L., Daniels, M., \&Yen, H., (2001). The Role of Vision Sensors in Future Intelligent Vehicles. Society of Automotive Engineers Technical paper 2001-01-2517

Hoare, E. G., Hall, P. S., Hill, R., Tsang, S. H., Thompson, C., Fu, S., \& Clarke, N., (2002) Millimetre-Wave Automotive Radar Advance Path Measurement Society of Automotive Engineers Technical paper 2002-01-0820

Lee, s., Kwon, W., \& Lee, J. W., (1999) A vision based lane departure warning system http://user.chollian.net/ robust87/paper/Iros99.pdf

Moray, N. 2004, "Ou' sont les neiges d'antan?". In D. A. Vincenzi, M. Mouloua, \& P. A. Hancock (Eds.), Human Performance, Situation Awareness and Automation; Current Research and Trends. (Mahwah, NJ: LEA).

Naikar N, Hopcroft R, \& Moylan A (2005) Work Domain Analysis: Theoretical Concepts and Methodology. DSTO-TR1665

National Highway Traffic Safety Administration (1999) Run-Off-Road Collision Avoidance Using IVHS Countermeasures

National Safety Council. (2002) Accident Facts: Deaths due to Motor-Vehicle Accidents, IN: National Safety Council Online Accident Facts. September 26, 1997.

Norman, D. A., (1990), The problem with automation: inappropriate feedback and interaction, not 'over-automation', in D.E Broadbent, J. Reason and A. Braddeley (eds), Human Factors in Hazardous Situations (Oxford: Oxford Science Publications)

Pohl, J., \& Ekmark, J., (2003) Development of a Haptic Intervention System for Unintended Lane Departure. Society of Automotive Engineers Technical paper 2003-01-0282

Prakah-Asante, K. O., Rao, M. K., \& Strumolo, G. S., (2003). Obstacle State Estimation For Imminent Crash Prediction \& Countermeasure Deployment Decision-Making . Society of Automotive Engineers Technical paper 2003-01-2261

Prakah-Asante, K. O., Rao, M. K., \& Strumolo, G. S., (2002). Multi-Level Sensing and Situation Awareness Evaluation for Adaptive Collision Countermeasure Activation. Society of Automotive Engineers Technical paper 2002-01-1883

Reason, J. (1990). Human error. Cambridge University Press, Cambridge.

ROSPA (2001) Driver Fatigue and Road Accidents - A Literature Review and Position Paper. Birmingham: The Royal Society for the Prevention of Accidents. 
Rudin-Brown, Ch. M.; Noy, Y. I., (2002) Investigation of behavioral adaptation to lane departure warnings. Transportation Research Record, no. 1803, pp. 30-37. 2002

Suzuki, K., Jansson, H., (2003) An analysis of driver's steering behaviour during auditory or haptic warnings for the designing of lane departure warning system. JSAE Review 24 (2003) 65-70

Tilin, A., (2002) You Are About To Crash, Wired Magazine Issue 10.04 http://www.wired.com/wired/archive/10.04/driver.html

The highway code. (1993). London: HMSO.

Tuffano, D. R. (1997) Automotive HUDs: The overlooked safety issues. Human Factors, 39(2), 303-311.

Vicente KJ (1999). Cognitive work analysis: Toward safe, productive, and healthy computer-based work. Mahwah, NJ.

Wallace, J. S.; Fisher, D. L. \& Collura, J. (1996) Sound localisation: Information Theory Analysis. Proceedings of the Human Factors and Ergonomics Society 40thAnnual Meeting (pp 905-909). Santa Monica: HFES.

Wheatley, D. J., (2000) The Driving Need for Human Factors in the Car of the Future, Society of Automotive Engineers Technical paper 2000-01-3075

Ziegler, W., et al. (1995) Computer Vision on the Road: A Lane Departure and Drowsy Driver Warning System. Society of Automotive Engineers Technical paper 952256 